\title{
Os Impactos da implantação de um bundle de prevenção de pneumonia associada à ventilação mecânica numa unidade de terapia intensiva no interior de Rondônia
}

\author{
The impacts of the implantation of a pneumonia prevention bundle associated with \\ mechanical ventilation in an intensive care unit inside Rondônia
}

Los impactos de la implantación de un bundle de prevención de neumonía asociado con

la ventilación mecánica en una unidad de cuidados intensivos dentro de Rondônia

Állef Diego Bonfim de Andrade ${ }^{1 *}$, Leiri Bonet ${ }^{1}$, Angela Antunes de Morais Lima ${ }^{1}$, Olavo Silva Valente $^{1}$, Ingryd Raiany Silva de Oliveira ${ }^{1}$, Erick Marques Pinheiro ${ }^{2}$, Maycon Pelosato Duarte ${ }^{2}$, Valério Bortolini ${ }^{2}$.

\section{RESUMO}

Objetivo: Reconhecer e apontar os índices de Pneumonia Associada à Ventilação Mecânica (PAVM) anteriormente e após a implementação de um bundle de prevenção de PAVM em uma unidade de terapia intensiva no interior do estado de Rondônia. Métodos: Foi realizado um estudo na modalidade transversal, retrospectiva e de abordagem quantitativa. As informações e dados foram levantados através de uma análise dos resultados encontrados após a implementação do bundle prevenção de PAVM. Durante a pesquisa, foram coletados dados referentes a todo o ano de 2017 (antes da implementação do bundle) e do mês de junho de 2018 ao mês de maio de 2019 (após da implementação do bundle). Os resultados da densidade de incidência foram calculados ao final dos 12 meses de cada período analisado. A extração e análise dos dados foram executadas com apoio do extranet do Institute for Healthcare. Resultados: Constamos que a elevação de $13,72 \%$ no uso de Ventilação Mecânica dia (VM/dia), porém ao mesmo tempo ocorreu uma queda de 1,1\% densidade de incidência após da implantação do bundle. Conclusão: Portanto, mesmo havendo um aumento na taxa de uso da ventilação mecânica, as ações de implementação do bundle refletiu na redução da densidade de incidência durante o período estudado.

Palavras-chave: Ventilação mecânica invasiva, Pneumonia, Fisioterapia, Unidade de terapia intensiva.

\begin{abstract}
Objective: To recognize and point out the rates of Pneumonia Associated with Mechanical Ventilation (VAP) before and after the implementation of a VAP prevention bundle in an intensive care unit in the interior of the state of Rondônia. Methods: A cross-sectional, retrospective and quantitative study was carried out. The information and data were collected through an analysis of the results found after the implementation of the VAP prevention bundle. During the survey, data were collected for the whole of 2017 (before the implementation of the bundle) and from June 2018 to May 2019 (after the implementation of the bundle). The results of the incidence density were calculated at the end of the 12 months of each analyzed period. Data extraction and analysis were performed with support from the Institute for Healthcare extranet. Results: We found that the increase of $13.72 \%$ in the use of Mechanical Ventilation day (MV / day), however at the same time there was a drop of $1.1 \%$ incidence density after the implantation of the bundle. Conclusion: Therefore, even with an increase in the rate of use of mechanical ventilation, the actions to implement the bundle reflected in the reduction of the incidence density during the period studied.
\end{abstract}

Keywords: Invasive mechanical ventilation, Pneumonia, Physiotherapy, Intensive care unit.

${ }^{1}$ Hospital Regional de Cacoal (HRC), Cacoal - RO. *E-mail: allefdiego_bonfim@hotmail.com

${ }^{2}$ Hospital de Urgência e Emergência de Rondônia (HEURO), Cacoal - RO.

SUBMETIDO EM: 11/2019

ACEITO EM: 12/2019

PUBLICADO EM: 2/2021

REAS/EJCH | Vol.13(2) | e2328 | DOI: https://doi.org/10.25248/reas.e2328.2021 Página 1 de 8 


\section{RESUMEN}

Objetivo: Reconocer y señalar las tasas de Neumonía Asociada a Ventilación Mecánica (NAV) antes y después de la implementación de un paquete de prevención NAV en una unidad de cuidados intensivos del interior del estado de Rondônia. Métodos: Se realizó un estudio transversal, retrospectivo y cuantitativo. La información y los datos fueron recolectados a través de un análisis de los resultados encontrados luego de la implementación del paquete de prevención de NAV. Durante la encuesta, se recopilaron datos para todo 2017 (antes de la implementación del paquete) y de junio de 2018 a mayo de 2019 (después de la implementación del paquete). Los resultados de la densidad de incidencia se calcularon al final de los 12 meses de cada período analizado. La extracción y el análisis de datos se realizaron con el apoyo de la extranet del Institute for Healthcare. Resultados: Encontramos que el aumento del 13,72\% en el uso de Ventilación Mecánica día (VM / día), sin embargo, al mismo tiempo hubo una caída de la densidad de incidencia del 1,1\% después de la implantación del haz. Conclusión: Por tanto, incluso con un aumento en la tasa de uso de ventilación mecánica, las acciones para implementar el paquete se reflejaron en la reducción de la densidad de incidencia durante el período estudiado.

Palabras clave: Ventilación mecánica invasiva, Neumonía, Fisioterapia, Unidad de cuidados intensivos.

\section{INTRODUÇÃO}

As infecções de origem nosocomial estão entre as principais causas de mortalidade dos pacientes graves internados nas Unidades de Terapia Intensiva (UTI), sendo que as infecções mais frequentes são as urinárias, as feridas cirúrgicas e as pneumonias. Pacientes com redução da capacidade respiratória e metabólica utilizam a ventilação mecânica invasiva (VMI), que é um método de ventilação artificial que permite a garantia da manutenção das trocas gasosas importantes para bom funcionamento do nosso organismo, considerado um suporte terapêutico habitualmente utilizado nas unidades de terapia intensiva (UTI), porém que expõe os doentes ao risco de adquirir pneumonia associada à ventilação mecânica (PAVM) (DALMORA CH, et. al., 2013).

Através de uma revisão bibliográfica Correa RA, et al. (2018), encontraram as seguintes definições: a pneumonia é definida como presença de novo infiltrado pulmonar mais indícios clínicos que sinalizem que o infiltrado é de origem infecciosa, incluindo novos episódios de febre, expectoração com secreção purulenta, leucocitose e diminuição da oxigenação, já a PAVM é definida como a pneumonia que inicia após 48 horas de intubação endotraqueal.

Segundo Dalmora $\mathrm{CH}$, et al., (2013), o desenvolvimento de pneumonias no ambiente hospitalar e de terapia intensiva, principalmente pneumonias associadas à ventilação mecânica, apresenta morbidade associada significativa, prolongando o tempo de ventilação mecânica e o tempo de permanência na UTI, todos custos associados a essa extensão.

O diagnóstico de pneumonia relacionada à ventilação mecânica à beira do leito deve levar em consideração os resultados abrangentes dos exames clínicos, de imagem e laboratoriais. Tendo em vista a baixa especificidade dos critérios clínicos isoladamente, os dados microbiológicos são usados como uma tentativa de melhorar a precisão do diagnóstico. Esses critérios incluem: se há uma infiltração contínua nova ou rogressiva, fusão ou cavitação; e pelo menos os dois critérios a seguir: febre (temperatura axilar acima de $38^{\circ} \mathrm{C}$ ), nenhuma outra causa ou leucopenia (12.000 células / $\mathrm{mm} 3$ ), ou o aparecimento de secreção purulenta ou mudanças nas características de descarga ou aumento da descarga. A PAVM e suas interfaces ainda causam impacto sobre a evolução clínica dos sujeitos principalmente quanto ao fator tempo de ventilação mecânica e de internação na UTI. A maior incidência de óbito na UTI ocorre nas primeiras semanas (VIANA AA, et al., 2018). Uma pesquisa chegou ao resultado que o tubo orotraqueal atua como desencadeador da PAVM pela formação do biofilme na sua superfície, possibilitando o aumento da patogênese da infecção.

Ainda, o contato com a camada microbiana dentro do biofilme pode contribuir para a patogênese da PAVM e promover um impacto na terapia antimicrobiana, elevando as taxas de morbimortalidade associadas a este tipo infecção (LIMA JLC, et al., 2017). 
Baseados em evidências científicas anteriores o Institute for Healthcare Improvement (IHI) (2008) concluiu que PAVM está relacionada aos cuidados de saúde e que geralmente são causados por inalação, a principal fonte são as secreções do trato respiratório superior e, a seguir, a inoculação externa de substâncias contaminadas ou refluxo gastrointestinal.

Estas aspirações são, mais comumente, microaspirações silenciosas, raramente há macroaspirações, que quando acontecem trazem um quadro de insuficiência respiratória grave e rapidamente progressiva. Além elevação da mortalidade, a repercussão desta infecção, especialmente da PAVM, traduz-se no prolongamento da hospitalização, em torno de 12 dias e no aumento de custos, em torno de 40000 dólares por episódio. Segundo a IHI (2012), é um grupo de intervenções baseadas em evidências científicas que quando adotadas juntas resultam em melhores resultados do que adotadas individualmente.

O conceito dos bundles teve início em 2001, porém passou a ser amplamente difundido a partir de 2004 com a campanha para salvar cem mil vidas, lançada pela organização não governamental americana Institute for Healthcare Improvement (BERWICK DM, et al., 2006).

No Brasil, a mortalidade associada aos eventos adversos está entre a $1^{\text {a }}$ à $5^{\text {a }}$ causas de óbito, com 104.187 a 434.112 possíveis mortes e $R \$ 15,5$ bilhões gastos anualmente pela insegurança assistencial hospitalar (COUTO RC, et al., 2016). Num estudo mais recente no ano de 2012 o IHI foi proposto um guia de como prevenir a PAVM sugerindo um bundle de ações que quando realizadas em conjunto podem reduzir os índices de infecções em UTIs.

Por ser um grande problema de saúde pública que por sua vez está mais associado aos atendimentos de alta complexidade como em UTIs, a prevenção e combate à PAVM é um desafio enorme para o SUS, pois a mesma gera benefícios para o paciente e torna visível a melhora na qualidade de assistência em saúde, por esse motivo existem vários projetos e programas que visão promover essa prevenção sendo implantados em todo o país (BRASIL, 2017)

Esta pesquisa se justificou pela necessidade de levantamento de dados que comprovem a possível eficácia da implantação de bundle de prevenção e controle de infecção em UTIs, visando promover maior valorização dessa ferramenta e ainda expor esses dados à comunidade local e científica para que os mesmos tenham ciência das melhorias na prestação da assistência.

Através da problemática apresentada, o estudo teve o seguinte objetivo: identificar e apontar os índices de Pneumonia Associada à Ventilação Mecânica antes e após a implantação de um bundle de cuidados e prevenção de Pneumonia Associada à Ventilação Mecânica em uma unidade de terapia intensiva na região central do estado de Rondônia.

\section{MÉTODOS}

O presente estudo foi elaborado por meio de uma pesquisa transversal, de caráter descritivo, retrospectivo, com abordagem quantitativa tendo o levantamento de dados estatísticos coletados juntos a equipe da Comissão de Controle de Infecção Hospitalar e do Projeto "Melhorando a Segurança do Paciente no Brasil em Larga Escala".

A população de estudo e amostra foram dos prontuários de pacientes que foram internados na UTI1 adulto submetidos à intubação endotraqueal por mais de 48 horas no período 12 meses antes a implantação do bundle de prevenção e PAVM (janeiro a dezembro de 2017) e 12 meses após a implementação do bundle de prevenção de PAVM (junho de 2018 a maio de 2019).

Os critérios de exclusão foram dados de prontuários de pacientes que não fazem parte do período da pesquisa e que possuem dados incompletos. Pesquisa foi elaborada após a aprovação do Comitê de Ética e Pesquisa (CEP) com o protocolo: 3.201.223.

Tendo o levantamento de dados elaborado através de três fases: a primeira foi realizada por um levantamento através dos dados estatísticos da Comissão de Controle de Infecções Hospitalares $(\mathrm{CIH})$ referentes aos índices de PAVM no período de Janeiro de 2017 a Dezembro de 2017. Na segunda fase foram 
colhidos os dados apresentados pelo Projeto "Melhorando a Segurança do Paciente no Brasil em Larga Escala" com a implantação do bundle de prevenção de PAVM contabilizando o índice de PAVM no período de junho de 2018 a Maio de 2019. Na terceira e última etapa foi utilizada a cálculo estatístico da Densidade de Incidência como instrumento para fazer a comparação dos índices de PAVM antes e após a utilização do bundle de prevenção de PAVM. A fórmula utilizada foi a seguinte:

$$
D i=\frac{N^{o} P A V M / M \text { ês }}{N^{o} \text { VM Dia/Mês }} X(1000)
$$

Para análise estatística, as variáveis categóricas foram expressas por valores brutos, ventilação mecânica/dia e a densidade de incidência. As médias foram realizadas por meio de estatística descritiva. Para comparação dos dados utilizou-se o como apoio do extranet do Institute for Healthcare Improvement (IHI).

\section{RESULTADOS}

\section{Primeira fase da pesquisa}

Ao final, a amostra pré-intervenção que gerou um resultado em 2017 a densidade de incidência de PAVM foi de 8,29 com 1678 pacientes com VM/dia, do qual 14 deles que foram diagnosticados com PAVM conforme a Tabela 1, que demonstrou uma Densidade de Incidência de PAVM de 8,29.

Tabela 1 - Dados da CCHI entre janeiro de 2017 a dezembro 2017.

\begin{tabular}{cccccccccccccc}
\hline Meses analisados & Jan & Fer & Mar & Abr & Mai & Jun & Jul & Ago & Set & Out & Nov & Dez & Total \\
\hline $\begin{array}{c}\text { № de pacientes dia na } \\
\text { UTI }\end{array}$ & 294 & 305 & 305 & 308 & 289 & 294 & 294 & 304 & 283 & 258 & 271 & 300 & 3505 \\
\hline $\begin{array}{c}\text { № de Ventilação } \\
\text { Mecânica dia UTI }\end{array}$ & 175 & 145 & 138 & 167 & 140 & 122 & 148 & 196 & 100 & 105 & 123 & 119 & 1678 \\
\hline $\begin{array}{c}\text { № de ocorrência de } \\
\text { PAVM }\end{array}$ & 1 & 2 & 0 & 0 & 2 & 2 & 3 & 1 & 0 & 0 & 1 & 2 & 14 \\
\hline
\end{tabular}

Fonte: Andrade ADB, et al., 2019.

\section{Segunda fase da pesquisa}

Ao final, a amostra pós-intervenção gerou um resultado entre maio de 2018 e junho de 2019 de 8,20 com 1945 pacientes com VM/dia, do qual 16 deles que foram diagnosticados com PAVM, o que conforme a Tabela 2, que demonstrou uma Densidade de Incidência de PAVM de 8,20.

Tabela 2 - Dados da CCHI entre junho de 2018 a maio 2019.

\begin{tabular}{cccccccccccccc}
\hline Meses analisados & Jun & Jul & Ago & Set & Out & Nov & Dez & Jan & Fev & Mar & Abr & Mai & Total \\
\hline $\begin{array}{c}\text { № de pacientes dia } \\
\text { na UTI }\end{array}$ & 285 & 289 & 291 & 282 & 285 & 279 & 251 & 263 & 435 & 302 & 276 & 253 & 3491 \\
\hline $\begin{array}{c}\text { № de Ventilação } \\
\text { Mecânica dia UTI }\end{array}$ & 226 & 144 & 122 & 168 & 147 & 165 & 166 & 156 & 120 & 191 & 121 & 219 & 1945 \\
\hline $\begin{array}{c}\text { № de ocorrência de } \\
\text { PAVM }\end{array}$ & 3 & 1 & 1 & 1 & 2 & 2 & 1 & 0 & 0 & 2 & 2 & 1 & 16 \\
\hline
\end{tabular}

Fonte: Andrade ADB, et al., 2019.

\section{Terceira fase}

Após dados os dados e cálculos das fases anteriores, fez-se a comparação dos índices de PAVM antes e após a utilização do bundle de prevenção de PAVM. Os resultados demonstram que houve uma elevação de $13,72 \%$ no uso de Ventilação Mecânica (VM) por dia, porém ao mesmo tempo houve uma queda de 1,1\% densidade de incidência depois da implementação do bundle de prevenção de PAVM. 


\section{DISCUSSÃO}

Não foi identificada a diminuição no número bruto de PAVM na UTI adulto do Hospital após a implantação do bundle, porém houve um aumento da exposição de pacientes ao risco identificado pelo um aumento na taxa de uso da ventilação mecânica, mas o processo de implementação do mesmo, refletiu na diminuição na densidade de incidência durante o período avaliado.

Uma das premissas dos estudos em saúde, assim como o nosso, é promover uma melhora na assistência com o intuito da redução da mortalidade, a Agência Nacional de Vigilância Sanitária (ANVISA) afirmou que a taxa de mortalidade geral de episódios de pneumonia relacionados à VM varia de $20 \%$ a $60 \%$, o que reflete amplamente a gravidade da doença subjacente nesses pacientes, a falência de órgãos e a especificidade da população estudada e os patógenos envolvidos (BRASIL, 2017). Em diferentes estudos, estima-se que a mortalidade por essa infecção seja diferente, mas aproximadamente $33 \%$ dos pacientes com PAVM morrem de infecção direta. Quando os indicadores relacionados à infecção foram coletados sistematicamente, a incidência de PAVM diminuiu após a adoção de medidas preventivas, indicando que PAV e pneumonia que podem não estar relacionadas à ventilação mecânica são complicações evitáveis.

As Infecções Relacionadas à Saúde (IRAS) constituem num alto risco à saúde dos usuários e, desse modo, sua prevenção e controle são de grande importância e envolvem medidas de qualificação da assistência hospitalar, por meio de ações que resultem na melhoria da qualidade da assistência à saúde, diminuem esforços, complicações e recursos.

Os serviços de terapia intensiva são unidades prioritárias para o desenvolvimento e implantação de indicadores de qualidade, devido à demanda de muitos processos desenvolvidos em pacientes críticos e que, em grande parte, dependem exclusivamente dos cuidados da equipe para sobreviver durante o período de internação (SILVA LTR, et al., 2011). Por esse motivo se fazem necessárias várias discussões para os achados deste estudo que foram estrategicamente organizados nas seguintes categorias:

\section{Medidas de prevenção de PAVM}

A PVMA continua sendo uma situação clínica significativa, com taxas de incidência relatadas de $7 \%$ a $15 \%$. Tendo em vista as graves consequências adversas associadas a esta infecção, a prevenção da PAVM tornou-se uma medida indispensável na maioria dos hospitais. Muitas instituições implantaram pacotes de prevenção eficazes de que combinam elevação da cabeceira da cama, higiene das mãos, higiene oral com clorexidina e drenagem subglótica, realizar o mais precoce possível testes de respiração espontânea e despertar diária mostraram consistentemente a redução na duração da ventilação mecânica pois de modo secundariamente isso também previne a PAVM, Dados mais recentes demonstram o impacto geral positivo dos bundles de prevenção, incluindo algumas de suas intervenções de componentes principais (VAZQUEZ GC e KOLLEF MH, 2018).

No hospital do estudo, o bundle de prevenção da PVAM implantado seguiu as recomendações da Agência Nacional de Vigilância Sanitária (ANVISA) que são as seguintes: cuidados e medidas para: manter decúbito elevado (30-45), Ajuste diário do nível de sedação e teste de respiração espontânea, sucção de rotina de secreções subglóticas, higiene oral com desinfetantes, uso cuidadoso de bloqueadores neuromusculares, preferência por ventilação mecânica não invasiva, cuidados com o circuito do ventilador, cuidados com os umidificadores, cuidados com o sistema de aspiração, evitar extubação não programada (acidental) e reintubação, monitoração contínua da pressão de cuff, dar preferência a intubação orotraqueal, atenção com inaladores e cuidado com nebulizadores, sonda enteral na posição gástrica ou pos-pilorica, processamento de produto de assistência respiratória e cuidados gerais com outros dispositivos ligados a ventilação mecânica (BRASIL, 2017).

A PAVM é uma complicação potencialmente séria nesses pacientes que já estão gravemente enfermos. Os cuidados de higiene oral com clorexidina, escova de dentes ou combinação, junto com aspiração de secreções, podem reduzir o risco de PAVM nesses pacientes (HUA F, et al., 2016).

Ações e medidas ligadas indiretamente à prevenção da infecção são tão importantes quanto as mesmas, pois a redução de sedação, realização de um despertar diário e desmame ventilatório precoce, promove um 
menor tempo do paciente na ventilação mecânica, portanto tem menor exposição diminuindo assim os riscos de desenvolver uma PAVM (WIP C e NAPOLITANO L, 2009).

Outras estratégias que podem ser adotas é as condições associadas à redução do tempo de ventilação mecânica, que indiretamente previnem PAVM, bem como o manejo correta da ventilação mecânica de modo protetor, estratégia ventilatória protetora $(\mathrm{VC}=6 \mathrm{ml} / \mathrm{kg}$ peso predito pela estatura) visando manter Pressão Parcial de Dióxido de Carbono (PaCO2) entre 35 e $45 \mathrm{mmHg}$, associado a níveis de Pressão Positiva ao Final da Expiração (PEEP) suficiente para garantir uma adequada troca gasosa, com modo ventilatório Ventilação com Volume Controlado (VCV) ou Ventilação com Pressão Controlada (PCV).

Assim que possível, deve-se passar a modos assistidos ou espontâneos com o objetivo de adiantar a retirada da VM. E por fim a mobilização precoce que está ligada diretamente a redução do tempo de ventilação mecânica (BRASIL, 2017). Algumas medidas avançadas propostas para reduzir as taxas de PAVM são apoiadas por evidências sólidas como as citadas anteriormente, porém estão em estudos promissores sobre o uso da nutrição administração de probióticos são promissores, mas não recomendados porque ainda faltam estudos maiores.

Já outras técnicas como simulação de tosse, utilização do sistema de aspiração fechado ou até mesmo o uso de têxteis impregnados com óxido de cobre requerem mais investigações para comprovação da eficácia das mesmas. No entanto, mesmo medidas de eficácia comprovada ainda não é amplamente utilizada, provavelmente porque a mortalidade não é afetada ou por não haver uma clara vantagem de custo-benefício no cuidado do paciente crítico (COPPADORO A, et al., 2019).

\section{O profissional de saúde e as ações de prevenção de PAVM}

Os profissionais de saúde são a peça chave para melhora dos índices, pois dele vem as ações diretas ao paciente que podem, tanto prevenir, como provocar a PAVM, por esse motivo Pombo CMN, et al. (2010) na intenção de avaliar profissionais de saúde, aplicou um questionário a 104 profissionais, Concluiu que, em geral, independentemente da categoria profissional, o conhecimento sobre a PAV e seus fatores de risco relacionados é regular e o preparo dos profissionais é inferior ao esperado, o que é preocupante em alguns casos.

Por fim, recomendam e fortalecem o desenvolvimento de uma recomendação educativa que norteie o trabalho dos profissionais de saúde da UTI e considere as estratégias necessárias para prevenir a PAV, pois só através do conhecimento teórico e prático é que os profissionais de saúde tomarão consciência de seu papel na tomada de decisão, resolução e execução do plano de ações para de munição e controle da doença.

Os resultados de uma revisão sistemática indicaram que a higiene oral promove uma redução dos resultados de PAV, em UTI pacientes submetidos à ventilação mecânica e ainda concluíram que os profissionais de saúde devem estar cientes dos potenciais benefícios associados a higiene oral a fim de reduzirem a incidência de PAVM e o número de bactérias orofaríngeas, portanto a equipe multiprofissional (ou seja, médicos, enfermeiros, técnicos de enfermagem, fisioterapeutas, dentistas, nutricionista e entre outros) devem ser continuamente instruídos e recebem orientações tanto prática ideal a higiene, quanto da importância da remoção adequada do biofilme dental e do uso da escovação dentária associada à higiene oral (CAMARGO L e SILVA SN, 2019)

Em um estudo em que avaliou os efeitos da implementação de um programa de educação sobre higienização das mãos e a adesão a essa prática entre profissionais de uma UTI. Estudo quase-experimental com séries temporais interrompidas conduzido ao longo de 12 meses: 5 meses antes do programa de educação (período basal), 2 meses durante a fase intensiva do programa (período de intervenção) e 5 meses durante a fase de manutenção do programa (período pós-intervenção).

Também foram avaliadas a duração da ventilação mecânica (VM), a incidência de pneumonia associada à ventilação mecânica (PAVM) em 28 e 60 dias e mortalidade nos mesmos momentos. Com base em 959 observações, houve um aumento nas taxas de adesão à higienização das mãos de $31,5 \%$ no período basal para $65,8 \%$ no período de intervenção e para $83,8 \%$ no período pós-intervenção, representando uma razão de prevalência 2,09 e 2,66 maior que o período basal, respectivamente $(p<0,001)$. 
Concluíram que o programa de educação em higienização das mãos aumentou a adesão a esse procedimento durante o período de monitorização, porém sem alterar as taxas de PAVM, a duração da VM e a mortalidade (ROMERO DMP, et al., 2019).

Já numa outra pesquisa que objetivou avaliar a qualidade da assistência à saúde realizada por Silva LTR, et al. (2011) avaliaram que: a assistência prestada em UTI, quanto ao uso das ações de prevenção e controle de pneumonia em pacientes de alto risco, submetidos a ventilação mecânica através de pesquisa descritiva exploratória, na qual foram realizadas 839 avaliações de pacientes em assistência ventilatória invasiva, no período de novembro de 2009 a janeiro de 2010, utilizando-se o Indicador de avaliação da adesão às medidas de prevenção e controle de pneumonia em pacientes de alto risco.

Algumas medidas isoladas que compõem o Indicador alcançaram índices próximos a 100\%, porém, o índice da conformidade total a todas as medidas de prevenção e controle de PAVM, correspondeu a 26,94\%. Ao final, concluíram que embora essas práticas avaliativas tenham sido desenvolvidas dentro da unidade, as intervenções ainda precisam ser avaliadas de forma sistemática para que a equipe de saúde possa discutir e implementar outras estratégias educativas. Por esse motivo que uma das justificativas deste trabalho é expor esses dados à comunidade local e científica para que eles tenham ciência das melhorias na prestação da assistência, podendo assim mudar suas práticas e melhorar seus resultados.

\section{Processos e cuidados}

Segundo Dalmora $\mathrm{CH}$, et al. (2013), afirmaram em seu estudo que o desenvolvimento das estratégias de prevenção ocorreu com a difusão dos seguintes conceitos: a implementação conjunta de um conjunto de medidas aumenta a probabilidade de cumprimento dessas medidas e seus efeitos na prevenção, neste caso os bundles. Alguns estudos que aplicaram esse conceito mostraram resultados positivos, reduzindo muito a incidência de infecções hospitalares potencialmente evitáveis e PAVM. Entretanto, a partir de um resultado positivo a diminuição das taxas de PAVM ocorridas com a aplicação de bundles, fez-se uma leitura equivocada: o conceito de que todo episódio de pneumonia ocorrido em um doente sob ventilação mecânica é um evento adverso que dá pra ser previnido.

Diferenças importantes na população avaliada foram ignoradas, bem como as características fisiopatogênicas da PAVM as formas e características distintas de outras infecções hospitalares. Hoje há uma visão de que o conceito de que a infecção hospitalar é um evento danoso ao paciente totalmente evitável e que a ocorrência ou não é utilizada como indicador da qualidade das assistências em saúde na UTI. Na realidade do hospital estudo, ele está inserido em um projeto nacional "Melhorando a Segurança do Paciente em Larga Escala do Brasil", o projeto é desenvolvido por meio do Proadi-SUS em parceria com o IHI que significa Instituto de Melhoria da Saúde. A meta nacional é salvar 8.500 vidas nas UTIs e reduzir em $\mathrm{R} \$ 1,2$ bilhão os custos decorrentes da infecção hospitalar no período de três anos e meio (BRASIL, 2017).

É medida também a adesão dos itens do bundle, o que não foi pesquisado nesse estudo, mas está diretamente ligado a redução dos índices de PAVM, como afirma O IHI (2012) que os hospitais que implantaram o bundle vêm encontrando resultados que relacionam as menores taxas de PAVM ao cumprimento de todos os itens do bundle alcançando uma redução de até $40 \%$. Porém os processos de adesão aos pacotes de prevenção apresentam uma demanda grande de gestão, ações e conscientização dos profissionais quanto ao uso adequado dessas práticas.

\section{CONCLUSÃO}

Os índices ao final da pesquisa foram o aumento de $13,72 \%$ no uso de Ventilação Mecânica (VM) por dia e a queda de $1,1 \%$ densidade de incidência após a implantação do bundle, o que não demonstra uma grande significância estatística, porém sugere o início de uma melhora nos resultados, porque a implantação das medidas de prevenção ainda eram recentes na UTI. Ao final, concluímos que mesmo sendo baixa a redução da densidade de incidência apresenta resultados positivos e ainda sugere que os processos de adesão ampla no ambiente hospitalar e que a gestão das medidas de prevenção seja essencial para a melhora dos índices em uma avaliação futura. 


\section{REFERÊNCIAS}

1. BRASIL. Agência Nacional de Vigilância Sanitária (ANVISA), Medidas de Prevenção de Infecção Relacionada à Assistência à Saúde. Brasília: ANVISA, 2017.

2. BRASIL. Ministério da Saúde (MS). Projeto "Melhorando a Segurança do Paciente em Larga Escala no Brasil", Brasília, 2017. Disponível em: http://portalarquivos2.saude.gov.br/images/pdf/2017/agosto/29/3-Forum-LatinoAmericano-de-Qualidade-e-Seguranca-na-Saude.pdf Acesso em: 08 de set 2019.

3. BERWICK DM, et al. The 100000 Lives Campaign: setting a goal and a deadline for improving health care quality. JAMA. 2006; 295(3):324-7

4. CAMARGO $L$, et al. Efficacy of toothbrushing procedures performed in intensive care units in reducing the risk of ventilatorassociated pneumonia: A systematic review. J Periodontal Res. 2019 Dec;54(6):601-611.

5. COPPADORO A, et al. Non-Pharmacological Interventions to Prevent Ventilator-Associated Pneumonia: A Literature Review. Respir Care. 2019;64(12):1586-1595.

6. COUTO RC, et al. Erros acontecem: a força da transparência no enfrentamento dos eventos adversos assistenciais em pacientes hospitalizados, Faculdade de Medicina da Universidade Federal de Minas Gerais (UFMG), 2016; 1(1): 458471 ,

7. CORREA RA, et al. Recomendações para o manejo da pneumonia adquirida na comunidade. J. bras. pneumol., 2018; 44(5): 405-423.

8. DALMORA CH, et. al. Definindo pneumonia associada à ventilação mecânica. Revista Brasileira de Terapia Intensiva, 2013; 25(2):81-86.

9. DAVID CMN. Infecção em UTI. Medicina (Ribeirão Preto, Online), 1998.

10. INSTITUTE FOR HEALTHCARE IMPROVEMENT (IHI). 5 million lives campaign. getting started kit: prevent ventilatorassociated pneumonia - how-to guide. Cambridge (Massachusetts): Institute for Healthcare Improvement; 2008.

11. INSTITUTE FOR HEALTHCARE IMPROVEMENT (IHI). How-to Guide: Prevent Ventilator-Associated Pneumonia. Cambridge (Massachusetts): Institute for Healthcare Improvement; Jan. 2012.

12. HUA F, et al. Oral hygiene care for critically ill patients to prevent ventilator-associated pneumonia. Cochrane Database Syst Rev., 2016; 25; 10(10): CD008367.

13. LIMA JLC, et al. Análise da produção de biofilme por isolados clínicos de Pseudomonas aeruginosa de pacientes com pneumonia associada à ventilação mecânica. Rev. Bras. Ter. Intensiva, 2017; 29(3): 310-316,

14. POMBO CMN, et al. Conhecimento dos profissionais de saúde na Unidade de Terapia Intensiva sobre prevenção de pneumonia associada à ventilação mecânica. Ciênc. Saúde coletiva, 2010; 15(supl. 1): 1061-1072.

15. ROMERO DMP, et al. Efeitos da implementação de um programa de educação de higienização das mãos entre profissionais de uma UTI: análise de séries temporais interrompidas. J. bras. pneumol., 2019; 45(5): e20180152.

16. SILVA SG, et al. PAV: discursos de profissionais acerca da prevenção. Esc Anna Nery, 2014;18(2):290-295

17. SILVA LTR, et al. Avaliação das medidas de prevenção e controle de pneumonia associada à ventilação mecânica. Rev. Latino-Am. Enfermagem, 2011; 19(6): 1329-1336.

18. VAZQUEZ GC, KOLLEF MH. Is Zero Ventilator-Associated Pneumonia Achievable?: Practical Approaches to Ventilator-Associated Pneumonia Prevention. Clin Chest Med. Dec 2018;39(4):809-822.

19. VIANA AA, et al. Clinical outcomes related to the incidence of ventilator-associated pneumonia in adults - a cohort study. Fisioterapia em movimento, 2018; 31: e 003115.

20. WIP C, NAPOLITANO L. Bundles to prevent ventilator-associated pneumonia: how valuable are they? Curr Opin Infect Dis., 2009; 22(2):159-66. 